\title{
Комбинированный способ
}

\author{
хирургического лечения
}

при паралитическом лагофтальме

с применением имплантатов

из политетрафторэтилена

И.А. Фикатова - s-р мед. наук, начальник отьела пластической хирургии

и глазного протезирования

С.А. Шеметов - аспирант отАела пластической хирургии и глазного протезирования

ФГБУ «Московский НИИ глазных болезней им. Гельмгольца» Минздрава России, 105062, Москва, ул. Садовая-Черногрязская, д. 14/19

Цель - разработка комбинированного способа устранения паралитического лагофтальма с применением имплантатов из политетрафторэтилена (ПТФЭ). Материал и методы. Клиническая группа - 20 пациентов, из них 8 мужчин, 12 женщин в возрасте 31-69 лет (44,7 \pm 5,3 года) с параличом лицевого нерва, пролеченных за последние 3 года. Срок от момента возникновения паралитического лагофтальма до начала хирургического лечения составил 1-9 лет (3,3 \pm 2,1 года). Описана типичная клиническая картина: лагофтальм - 9-16 мм

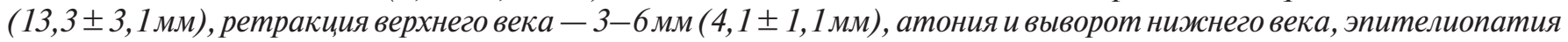

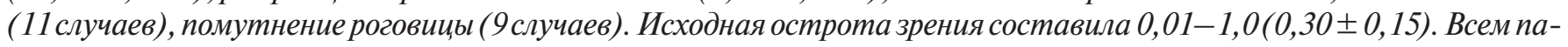
циентам проведено устранение лагофтальма с применением имплантата из ПТФЭ. Хирургическое вмешательство y 5 пациентов проведено в два этапа (отдельно на верхнем и нижнем веках), в 15 случаях - комбинированным способом одномоментно. В качестве имплантата использована лента из пористого ПТФЭтолщиной 200-300мкммонолитная или с перфорационными отверстиями. Срок наблюдения составил от 6 мес до 3 лет (1,9 0,9года). Результаты. В ранние и отдаленные сроки осложнений не выявлено. Увсех пациентов отмечена положительная динамика по критериям: величина лагофтальма, наличие выворота нижнего века, наличие ретракции верхнего века, состояние роговицы. Оценку проводили спустя б и более месяцев после операции. Во всех случаяхлагофтальм значительно уменьшился, остаточный лагофтальм составил 2-5 мм $(3,1 \pm 1,3$ мм). Состояние роговицы у пациентов с кератопатией улучшилось, зрение повысилось до 0,1-1,0 (0,50 \pm 0,25). Заключение. В предлагаемом способе лагофтальм устраняют как за счет укрепления нижнего века, так и за счет рецессии верхнего века, $а$ использование имплантатов из ПТФЭ обеспечивает стабильный эффект.

Ключевые слова: паралитический лагофтальм, хирургическое лечение, укрепление нижнего века, рецессия верхнего века, имплантаты из политетрафторэтилена.

Для цитирования: Филатова И.А., Шеметов С.А. Комбинированный способ хирургического лечения при паралитическом лагофтальме с применением имплантатов из политетрафторэтилена. Российский офтальмологический журнал. 2018; 11 (2): 27-34. doi: 10.21516/2072-0076-2018-11-2-27-34.

Одним из тяжелых состояний для наиболее чувствительной ткани глаза - роговицы является паралич век, развивающийся при поражении ветвей лицевого нерва, что в большинстве случаев приводит к развитию помутнений и язв роговицы [1-4]. Из-за нарушения иннервации круговой мышцы 
век развивается лагофтальм вследствие ретракции верхнего века, атонии и выворота нижнего века, что помимо снижения зрительных функций, субъективного дискомфорта из-за болевых ощущений и слезотечения значительно снижает качество жизни пациентов [1-4].

Для лечения этой тяжелой патологии век предложен ряд методов. Наиболее щадящий из них инъекции ботулотоксина А, его преимущество заключается в возможности выполнения в ранние сроки после нейрохирургических вмешательств [5-7], когда ожидается восстановление функций лицевого нерва или когда имеются противопоказания для проведения хирургического лечения. Однако в отдаленные сроки при уже сформировавшемся вывороте нижнего века данная методика малоэффективна.

Из хирургических способов широко распространены различные виды блефаро- и тарзорафии $[1,8,9]$. Применение тарзорафии и блефарорафии возможно в качестве временной или постоянной методики коррекции паралитического лагофтальма. Некровавая блефарорафия чаще выполняется по срочным показаниям как временная мера с целью защиты роговицы при остром процессе [8, 9].

Хотя эти органосохранные операции достаточно эффективны и позволяют значительно снизить проявления экспозиционной кератопатии, но их недостатками являются травматичность, ограничение поля зрения в некоторых случаях и плохой косметический результат [10].

Довольно часто при паралитическом лагофтальме прибегают к сужению глазной щели с помощью проведения различного типа нитей (силиконовой, аллонити, лески) через верхнее и нижнее веко с фиксацией ее концов в натянутом состоянии [1, 8-12]. Данные виды операций менее травматичны, и косметический результат несколько лучше, чем при проведении тарзорафии и блефарорафии, хотя полное открытие век в результате становится невозможным. Эта методика особенно актуальна у пожилых пациентов и у больных с длительно существующим параличом лицевого нерва, так как связки и фасции верхнего и нижнего века у таких пациентов истончаются и теряют свою прочность в связи с атрофическими процессами. Из осложнений этих операций отмечается прорезывание нити, а также развитие воспалительной реакции инфекционной и неинфекционной этиологии [10].

Уменьшение степени лагофтальма за счет опущения верхнего века выполняют с помощью удлинения леватора с применением различных трансплантатов или имплантатов. Довольно часто с этой целью применяют имплантацию ушного хряща, некоторые авторы используют широкую фасцию бедра [9, 11, 13]. Для удлинения верхнего века используют также кожно-клетчаточно-хрящевой лоскут. При этом лоскут (массой 0,9-1,4 г) фиксируют к тарзальной пластинке, а кожу лоскута сшивают с кожей века [9].
Однако, несмотря на удлинение верхнего века при данном способе и его дополнительное утяжеление за счет многослойности лоскута, недостатком данного способа является массивное рубцевание в зоне операции за счет той же многослойности лоскута, что в конечном итоге приводит к гипоэффекту и низкому косметическому эффекту. Описаны единичные случаи использования аллопланта для укрепления нижнего века [14].

Современная модификация операции утяжеления верхнего века заключается в использовании синтетического имплантата, который может быть выполнен из биосовместимого и биоинертного материала, из платины и/или из золота, с лекарственным покрытием, которое снабжено съемной защитной пленкой [15-19]. Однако данный способ не устраняет основную проблему паралитического лагофтальма - слабость и выворот нижнего века, а на верхнем веке в некоторых случаях контурируется имплантат в виде «плюс-ткани», что вызывает субъективный дискомфорт и снижает косметический эффект, описаны случаи обнажения и отторжения пальпебральных имплантатов [1, 10, 20, 21].

Одним из современных и малоинвазивных вмешательств является устранение лагофтальма, предусматривающее введение биодеградируемого препарата для инъекционной контурной пластики на основе гиалуроновой кислоты или коллагена общим объемом 0,25-4,0 мл, чаще в верхнее, реже в нижнее веко под претарзальную часть круговой мышцы и/или внутримышечно, и/или подкожно, и/или для верхнего века кпереди от сухожилия леватора [22-24]. Однако данная методика дает кратковременный эффект, и после рассасывания препарата (через несколько месяцев) возникает рецидив лагофтальма. Кроме того, данные инъекции дорогостоящие, а выполнять их приходится несколько раз в год.

В целом вышеуказанные методики приводят к опущению верхнего века и сужению глазной щели, но практически не влияют на положение нижнего века. Таким образом, проблема коррекции паралитического лагофтальма до настоящего времени остается актуальной, поскольку каждый из ранее применявшихся методов устраняет только часть имеющихся нарушений.

ЦЕЛЬЮ данной работы явилась разработка комбинированного способа устранения паралитического лагофтальма с применением имплантатов из политетрафторэтилена (ПТФЭ).

\section{МАТЕРИАЛ И МЕТОДЫ}

Клиническую группу составили 20 пациентов с параличом лицевого нерва, которым проведено хирургическое лечение в отделе пластической хирургии и глазного протезирования за период с 2014 по 2016 г. Возраст пациентов варьировал от 31 до 69 лет (в среднем 44,7 \pm 5,3 года), среди пациентов было 8 мужчин, 12 женщин. Причиной возникно- 
вения паралича лицевого нерва в 18 случаях было нейрохирургическое вмешательство в области мостомозжечкового угла (удаление невриномы слухового нерва, шванномы), удаление опухоли околоушной железы, в одном случае паралич развился в исходе перенесенной нейроинфекции, у одной пациентки паралич n. Facialis явился исходом черепно-мозговой травмы в результате ДТП. Срок, прошедший от момента возникновения паралитического лагофтальма до первого этапа хирургического лечения, варьировал от года до 9 лет (3,3 $\pm 2,1$ года).

Клиническая картина у всех пациентов была типичной (рис. 1, А, Б; 2, А, Б; 3, А, Б): лагофтальм от 9 до 16 мм (13,3 $\pm 3,1$ мм), ретракция верхнего века - 3-6 мм (4,1 $\pm 1,1$ мм), атония нижнего века, заднее ребро нижнего века не прилегало к поверх- ности глазного яблока за счет выворота нижнего века различной степени выраженности. Функция круговой мышцы отсутствовала полностью, подвижность глазного яблока сохранялась в полном объеме. На роговице в 11 случаях отмечалась точечная эпителиопатия, у 9 пациентов - локальные помутнения. Острота зрения варьировала от 0,01 до 1,0 $(0,30 \pm 0,15)$. Жалобы тоже были типичными: дискомфорт (рези, жжение, боли, слезотечение), снижение зрения, даже при vis $=1,0$ из-за слезотечения или использования мазей.

Всем пациентам было выполнено устранение лагофтальма с применением имплантата из ПТФЭ. Хирургическое вмешательство проведено у 5 пациентов в два этапа (отдельно на верхнем и нижнем веках), в 15 случаях - одномоментно комбинированным способом.
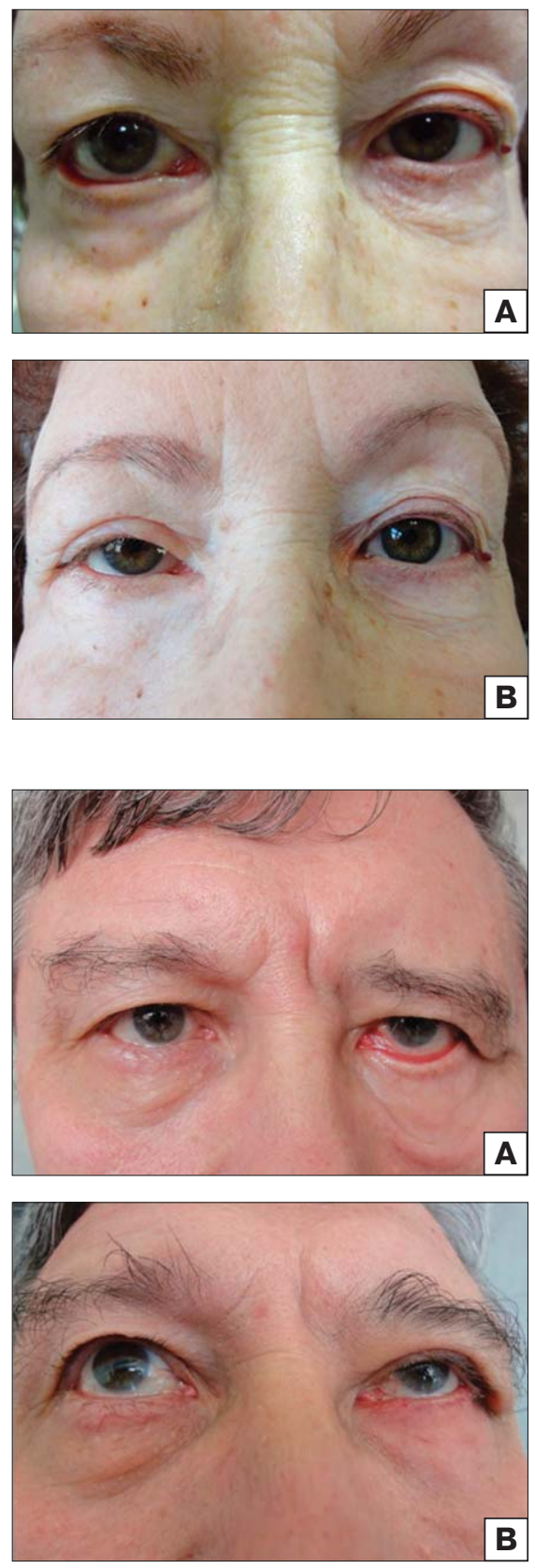
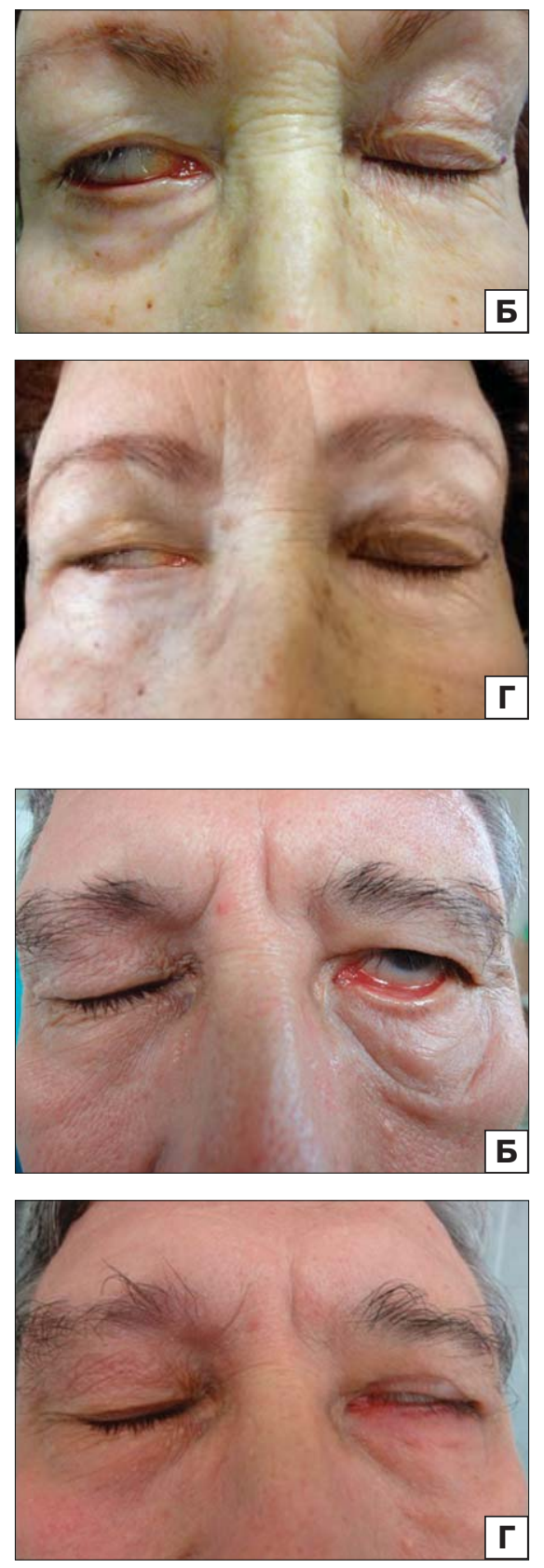

Рис. 1. Пациентка К. Паралитический лагофтальм в течение 5 лет после удаления невриномы слухового нерва. А, Б - до оперативного вмешательства; В, Г - через год после комбинированного вмешательства.

Fig. 1. Patient K. Paralytic lagophthalmos within 5 years after removal of VIII nerve neuroma. A, B - before surgery; B, Г a year after the combined surgery. 

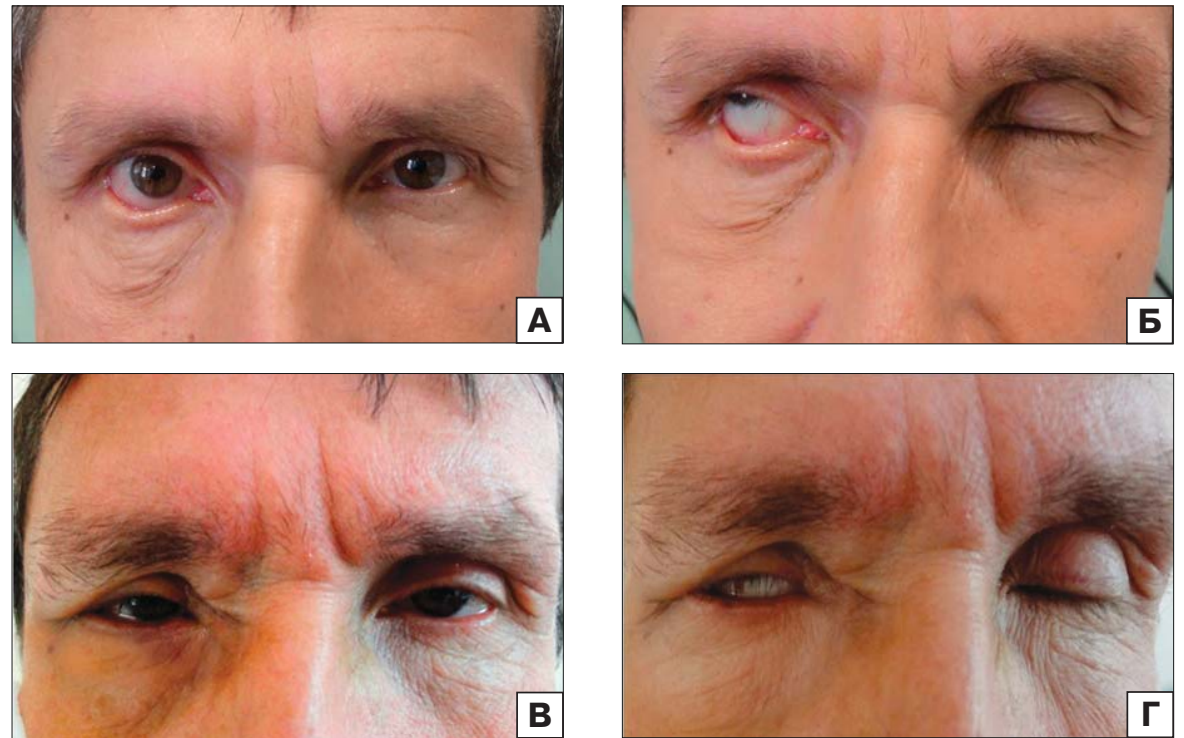

Рис. 3. Пациент Р. Паралитический лагофтальм в течение 5 лет после удаления невриномы слухового нерва. A, Б - до оперативного вмешательства; В, Г - через год после комбинированного вмешательства.

Fig. 3. Patient R. Paralytic lagophthalmos followed for 5 years after the removal of VIII nerve neuroma. A, B - before surgery; B, $\Gamma-$ a year after the combined surgery.
В качестве имплантационного материала использован имплантат в виде ленты из пористого ПТФЭ толщиной 200-300 мкм, монолитный или с перфорационными отверстиями диаметром 1,5 мм, расположенными в шахматном порядке с расстоянием между ними 3,5 мм (ЗАО НПК «ЭКОФЛОН», С.-Петербург, «Пленка пористая политетрафторэтиленовая для закрытия дефектов кровеносных сосудов», ТУ 9398-009-23167010-2010) [25].

Исследование проводили с разрешения локального этического комитета ФГБУ «Московский НИИ глазных болезней им. Гельмгольца» МЗ РФ. Все пациенты подписывали информированное согласие на проведение хирургического вмешательства.

Техника операции. Проводят разрез кожи нижнего века в 2-3 мм от линии ресниц, тупым путем в продольном направлении разделяют круговую мышцу. Дополнительно выполняют разрезы кожи на 5-7 мм выше проекции внутренней и наружной связок, от которых формируют тоннели в тканях к основному разрезу (рис. 4). Освобождают от окружающих тканей тарзальную пластинку и фиксируют имплантат из ПТФЭ к ее передней поверхности. Имплантат (монолитный или перфорированный) для укрепления нижнего века выкраивают шириной 6-7 мм и длиной 5-6 см. Концы имплантата проводят в сформированные тоннели (рис. 5) и фиксируют к связкам или к надкостнице в проекции связок век с натяжением. Дополнительно проводят разрез кожи верхнего века по складке, тупым путем разделяют волокна круговой мышцы. Далее производят рецессию леватора верхнего века с использованием имплантата из ПТФЭ, при этом апоневроз леватора отсекают от тарзальной пластинки и подшивают имплантат к тарзальной пластинке с одного края и к апоневрозу с другого края (рис. 6). Размер используемого имплантата: длина 20-25 мм, ширина от 5 до 10 мм в зависимости от желаемого эффекта рецессии. Накладывают отдельные швы на круговую мышцу, кожные раны ушивают узловыми или непрерывными швами. При перерастянутых атоничных веках дополнительно выполняют частичную наружную блефарорафию на 3-5 мм.

В послеоперационном периоде пациенты получали стандартную противовоспалительную терапию с применением кератопротекторов. Кожные швы снимали на 7-9-й день. Срок наблюдения за пациентами составил от 6 мес до 3 лет (1,9 \pm 0,9 года).

\section{РЕЗУЛЬТАТЫ}

Во всех случаях заживление операционных ран происходило в обычные сроки. Отек и подкожные кровоизлияния разрешились также в обычные сроки. У всех пациентов отмечена положительная динамика по критериям: величина лагофтальма, наличие выворота нижнего века, наличие ретракции верхнего века, состояние роговицы (см. рис. 1, В, Г; 2, В, Г; 3, В, Г). Оценку проводили спустя 6 и более месяцев после операции. Во всех случаях лагофтальм значительно уменьшился (рис. 7), остаточный лагофтальм варьировал от 2 до 5 мм ( $3,1 \pm 1,3$ мм), ретракции верхнего века и выворота нижнего века не было. Состояние роговицы у пациентов с кератопатией улучшилось, случаев эпителиопатии не отмечали. Острота зрения пациентов в отдаленные сроки после операции была от 0,1 до $1,0(0,50 \pm 0,25)$ (табл.).

Случаев неприживления (обнажения или отторжения) имплантатов не выявлено. Следует отметить, что субъективная оценка пациентов своего состояния значительно улучшилась, они отмечали повышение комфорта, снижение или отсутствие жжения, рези, боли. Рецидивов кератопатии в исследуемой группе не было отмечено.

\section{ОБСУЖДЕНИЕ}

В основе предлагаемого способа лежит идея сочетания различных хирургических подходов для устранения паралитического лагофтальма и 
Рис. 4. Схема оперативного вмешательства по предлагаемому способу. А - разрез кожи нижнего века в 2-3 мм от линии ресниц; Б - дополнительные разрезы в проекции наружной и внутренней связок, от которых сформированы тоннели, подготовлен имплантат из ПТФЭ необходимой формы; В - имплантат фиксирован к тарзальной пластинке, его концы проведены в подготовленные тоннели и фиксированы к связкам или надкостнице над связками; Г - послойное ушивание тканей над имплантатом, разрез кожи верхнего века по линии складки, мобилизация апоневроза леватора; Д - апоневроз леватора пересечен, имплантат из ПТФЭ подшит к тарзальной пластинке и к апоневрозу леватора; Е - раны ушиты узловыми и непрерывными швами.

Fig. 4. Surgery performed according to the proposed technique. A - skin incision of the lower eyelid $2-3 \mathrm{~mm}$ from the lash line; $\mathrm{B}$ - additional incisions in the projection of the medial and lateral ligaments, which are used to form tunnels, PTFE implant of the required form is prepared; $B$ - implant is fixed to the tarsal plate, its ends are brought to the tunnels prepared and fixed to the ligaments or to the periosteum over the ligaments; $\Gamma$ - layer-by-layer suturing of the tissues above the implant. Skin incision of the upper lid along the fold, mobilization of the aponeurosis of the levator; Д aponeurosis of the levator is crossed, PTFE implant is sutured to the tarsal plate and to the aponeurosis of the levator; E - the wounds are closed with knot and uninterrupted and sutures.

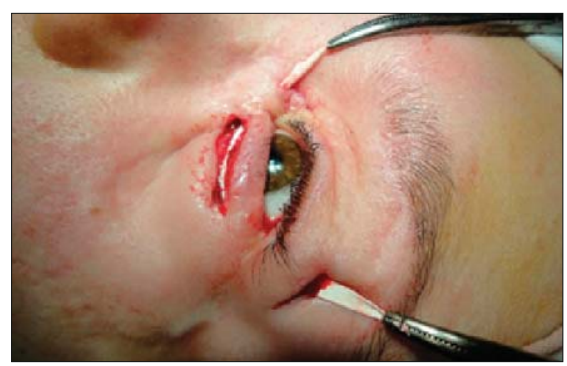

Рис. 5. Этап операции: имплантат из ПТФЭ фиксирован к тарзальной пластинке нижнего века, концы его проведены к связкам век.

Fig. 5. Surgery stage: the PTFE implant is fixed to the tarsal plate of the lower eyelid, the ends of the implant are brought to the ligaments of the eyelids.
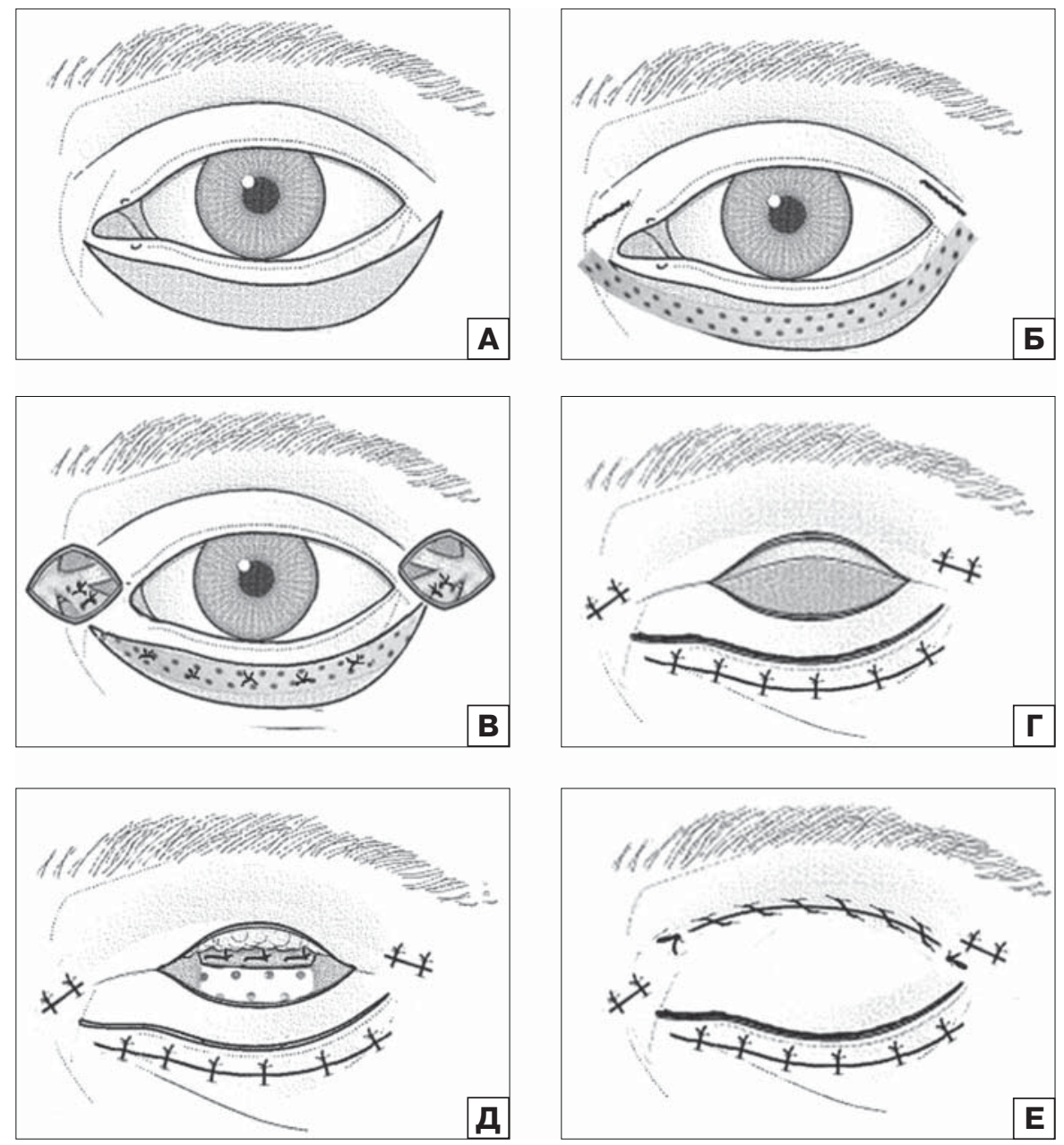

Рис. 6. Этап операции: имплантат из ПТФЭ подшит к тарзальной пластинке верхнего века и апоневрозу леватора.

Fig. 6. Surgery stage: the PTFE implant is sutured to the tarsal plate of the upper eyelid and to the levator aponeurosis.

Рис. 7. Величина лагофтальма до и после операции. Fig. 7. The amount of lagophthalmos before and after surgery.

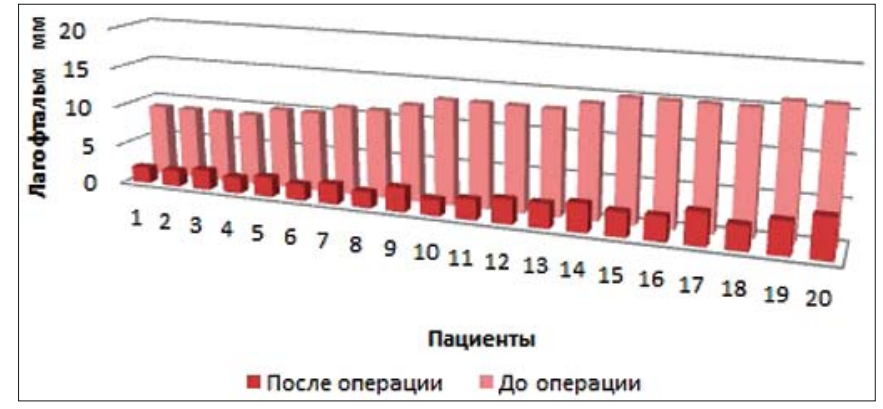


Таблица. Результаты хирургического лечения пациентов с паралитическим лагофтальмом комбинированным методом Table. Results of the surgical treatment of paralytic lagophthalmos using combined technique

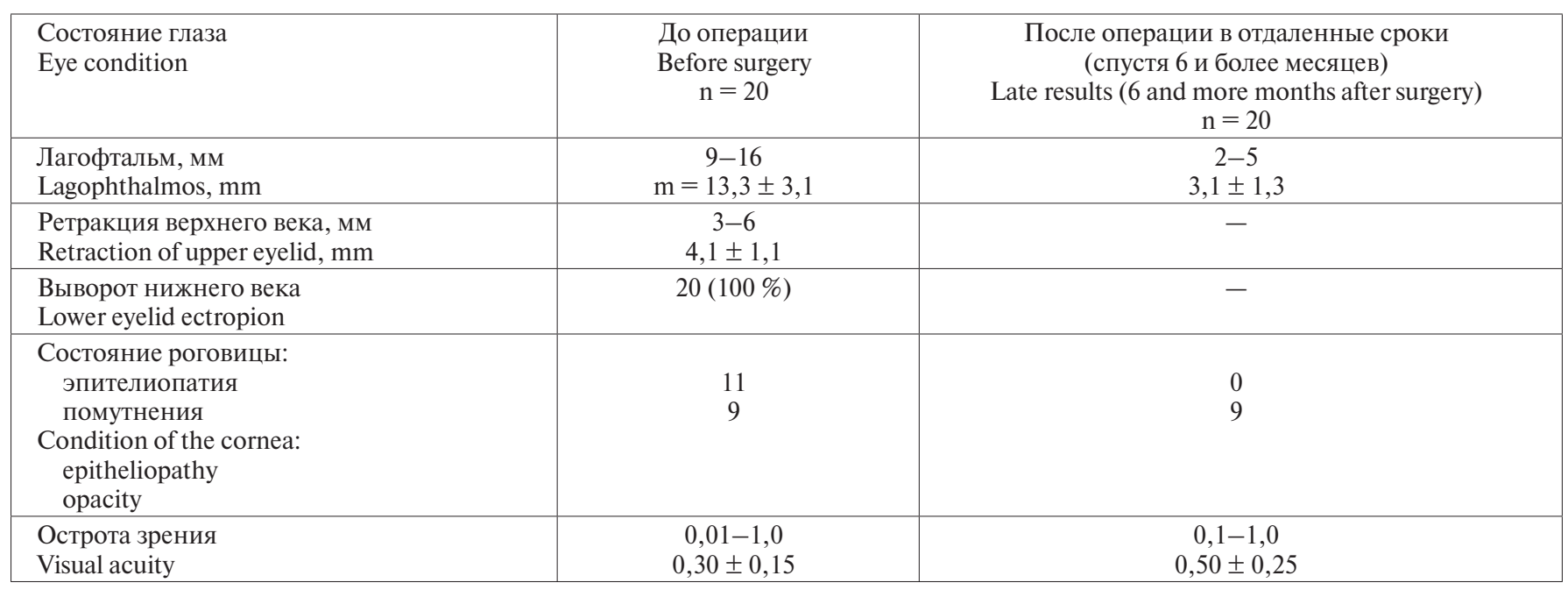

Примечание. $\mathrm{n}$ - количество пациентов.

Note. $n-$ number of patients.

использования в качестве имплантата материала из ПТФЭ. Толщина используемого имплантата 200-300 мкм обеспечивает мягкость и моделируемость имплантата при достаточной прочности. За счет используемой толщины имплантат не контурируется (не проминирует) на веках, при этом не ощущается пациентами. Использование пористого имплантата толщиной 200-300 мкм с перфорационными отверстиями способствует облегчению врастания окружающих тканей в толщу имплантата, что повышает стабильность нахождения имплантата в тканях как верхнего, так и нижнего века.

Этап укрепления нижнего века за счет его подтягивания за концы имплантата, фиксированного к передней поверхности тарзальной пластинки, и фиксации к надкостнице обеспечивает стабильный эффект и устраняет одномоментно лагофтальм и выворот нижнего века. То, что апоневроз леватора отсекают от тарзальной пластинки и подшивают имплантат к тарзальной пластинке с одного края и к апоневрозу с другого края, позволяет обеспечить уменьшение лагофтальма за счет удлинения мышцы, поднимающей верхнее веко, со стабильным эффектом. При перерастянутых и атоничных веках (в сочетании с возрастными изменениями) частичная наружная блефарорафия является дополнительным элементом стабильности для ослабленных век с нарушенной иннервацией. Кроме того, она усиливает положительный эффект за счет создания симметричного размера глазных щелей при перерастянутых атрофичных веках.

Результатом предложенного метода хирургического лечения при паралитическом лагофтальме является стабильность эффекта с отсутствием рецидивов и получением адекватного косметического результата.

\section{ЗАКЛЮЧЕНИЕ}

Таким образом, в предложенной комбинированной методике паралитический лагофтальм устраняют одномоментно как за счет укрепления нижнего века, так и за счет рецессии верхнего века, а использование имплантатов из ПТФЭ обеспечивает стабильный эффект, не ослабляющийся со временем, и высокий косметический результат.

\section{Конфликт интересов: отсутствует.}

Прозрачность финансовой деятельности: никТо из авторов не имеет финансовой заинтересованности в представленных материалах или методах.

\section{Лumepamypa}

1. McCord C.D. Eyelid Surgery. New York: Lippincott-Raven; 1995: 99-155.

2. Каспарова Е.A., Собкова О.И. Нейротрофический кератит на фоне паралитического лагофтальма. Офтальмология. 2009; $6(2): 11-4$.

3. Каспаров А.А., Каспарова Е.А., Собкова О.И. Лечение центральной язвы роговицы на фоне лагофтальма с помощью аутоконъюнктивальной пластики в сочетании с оптической иридэктомией и наружной блефарорафией. Вестник офтальмологии. 2010; 126 (2): 35-7.

4. Журова С.Г., Бржеский В.В., Калинина И.В., Ефимова Е.Л. Лечение язвы роговицы ксеротической этиологии. Клиническая офтальмология. 2010; 11 (2): 49-52.

5. Yücel O.E., Artürk N. Botulinum toxin-A-induced protective ptosis in the treatment of lagophthalmos associated with facial paralysis. Ophthal. Plast. Reconstr. Surg. 2012; 28 (4): 256-60. doi:10.1097/ IOP.0b013e31824ee702.

6. Табашникова Т.В., Серова Н.К., Шиманский В.Н., Григорьева H.Н. Применение ботулотоксина А (лантокс) для получения временного медикаментозного птоза у нейрохирургических больных. Вопросы нейрохирургии им. Н.Н. Бурденко. 2012; 4: 43-9.

7. Табашникова Т.В., Серова Н.К., Шиманский В.Н. Лечение трофической кератопатии и коррекция лагофтальма у нейрохирургических больных. Офтальмологические ведомости. 2013; 3: 9-16. 
8. Зайкова М.В. Пластическая офтальмохирургия. 2-е изд. Москва: Медицина; 1980.

9. Неробеев А.И., Шургая Ц.М., Мариничева И.Г. Методы коррекции паралитического лагофтальма. Анналы пластической, реконструктивной и эстетической хирургии. 1998; 2: 18-27.

10. Волков В.В., Бржеский В.В., Ушаков Н.А. Офтальмохирургия с использованием полимеров. С.-Петербург: Гиппократ; 2003.

11. Агафонова Е.И., Груша Я.О. Хирургические способы лечения паралитического лагофтальма. Вестник офтальмологии. 2010; 5: 48-52.

12. Сковородникова И.В. Способ коррекции лагофтальма при хроническом параличе лицевого нерва. Патент РФ № 2579351; 2016.

13. Pirrello R., D'Arpa S., Moschella F. Static treatment of paralytic lagophthalmos with autogenous tissues. Aesthetic Plast Surg. 2007; 31 (6): 725-31. doi:10.1007/s00266-007-0074-7.

14. Иволгина И.В. Блефаропластика лагофтальма и обширного выворота нижнего века при параличе лицевого нерва. Новое в офтальмологии. 2011; 2: 27-8.

15. Levin R., Shapiro J. Reanimation of the paralyzed eyelid with the enhanced palpebral spring or the gold weight: modern replacements for tarsorrhaphy. Facial Plast. Surg. 2000; 16: 325-36.

16. Bladen J.C., Norris J.H., Malhotra R. Cosmetic comparison of gold weight and platinum chain insertion in primary upper eyelid loading for lagophthalmos. Ophthal. Plast. Reconstr. Surg. 2012; 28 (3): 171-5. doi:10.1097/iop.0b013e3182467bf7.

17. Lessa S., Nanci M., Sebastiá R., Flores E. Treatment of paralytic lagophthalmos with gold weight implants covered by levator aponeurosis. Ophthal. Plast. Reconstr. Surg. 2009; 25 (3): 189-93. doi:10.1097/iop.0b013e3181c0e2e8.
18. Belsare $G$. Gold weights as upper eyelid implants or facial paralysis. Indian J. Otolaryngol. Head Neck Surg. 2010; 62 (1): 36-9.

19. Груша Я.О., Новиков И.А., Агафонова Е.И. Первый опыт применения имплантата для утяжеления верхнего века при паралитическом лагофтальме. Вестник офтальмологии. 2012; 1: 41-3.

20. Chepeha D.B., Yoo J., Birt C., Gilbert R.W., Chen J. Prospective evaluation of eyelid function with gold weight implant and lower eyelid shortening for facial paralysis. Arch. Otolaryngol. Head Neck Surg. 2001; 127 (3): 299-303. doi:10.1001/archotol.127.3.299.

21. Thomas D., Khalifa Y. Temporalis fascia in the management of gold eyelid weight extrusion. Ophthal. Plast. Reconstr. Surg. 2005; 21 (2): 153-5. doi:10.1097/01.iop.0000156499.84713.44.

22. Goldberg R.A., Lee S., Jayasundera T., et al. Treatment of lower eyelid retraction by expansion of the lower eyelid with hyaluronic acid gel. Ophthal. Plast. Reconstr. Surg. 2007; 23 (5): 343-8.

23. Mancini R., Taban M., Lowinger A., et al. Use of hyaluronic Acid gel in the management of paralytic lagophthalmos: the hyaluronic Acid gel "gold weight". Ophthal. Plast. Reconstr. Surg. 2009; 25 (1): 23-6. doi:10.1097/IOP.0b013e318192568d.

24. Груша Я.О., Исмаилова Д.С., Иванченко Ю.Ф., Агафонова Е.И. Первый опыт применения препарата стабилизированной гиалуроновой кислоты для коррекции лагофтальма. Вестник офтальмологии. 2010; 126 (1): 39-42.

25. Дьяков В.Е., Душенков А.С., Кортунов Ю.А. и др. Имплантат для восстановительной хирургии. Патент РФ № 166787; 2003.

Поступила: 11.07.2017

Combined method of surgical treatment for paralytic lagophthalmos with polytetrafluoroethylene (PTFE) implants

\author{
I.A. Filatova - Dr. Med. Sci., head of the department of plastic surgery and eye prosthetics \\ S.A. Shemetov - PhD student of the department of plastic surgery and eye prosthetics
}

\author{
Moscow Helmholtz Research Institute of Eye Diseases, 14/19, Sadovaya-Chernogryazskaya St., Moscow, \\ 105062, Russia \\ filatova64@yandex.ru
}

The purpose is to develop a combined method of paralytic lagophthalmos treatment using polytetrafluoroethylene (PTFE) implants. Material and methods. The clinical group consisted of 20 patients (8 males, 12 females) aged 31-69 (44.7 \pm 5.3 years) with facial nerve paralysis, who were treated within the last 3 years. The onset of paralytic lagophthalmos took place 1-9 years ( $3.3 \pm 2.1$ years) before surgical treatment. A typical clinical picture was as follows: lagophthalmos $9-16 \mathrm{~mm}(13.3 \pm 3.1 \mathrm{~mm})$, retraction of the upper eyelid $3-6 \mathrm{~mm}(4.1 \pm 1.1 \mathrm{~mm})$, atony and ectropion of the lower eyelid, epitheliopathy (11 cases), leucoma (9 cases). Visual acuity was 0.01-1.0 (0.3 \pm 0.15$)$. All patients had surgery for lagophthalmos with the use of a PTFE implant. In 5 patients, surgery was performed in two stages (separately for the upper and the lower eyelids), in 15 cases it was performed simultaneously, using a combined method The implant was a porous PTFE tape 200-300 $\mu \mathrm{m}$ thick, either solid or with perforations. The follow-up period was 6 months to 3 years ( $1.9 \pm 0.9$ years). Results. No complications have been revealed either in the early or late follow-up periods. All patients had positive dynamics of the following parameters: the size of lagophthalmos, the presence of ectropion of the lower eyelid, retraction of the upper eyelid, the condition of the cornea. The evaluation was conducted 6 months after surgery or later. In all cases, lagophthalmos decreased significantly, the residual lagophthalmos was 2-5 $\mathrm{mm}(3.1 \pm 1.3 \mathrm{~mm})$. The condition of the cornea in patients with keratopathy improved, vision increased to reach 0.1-1.0(0.5 \pm 0.25$)$. Conclusion. In the proposed method, the lagophthalmos is eliminated by lower eyelid strengthening and upper eyelid recession. PTFE implants provide a stable effect. 
Keywords: paralytic lagophthalmos surgical treatment, strengthening of the lower eyelid, recession of the upper eyelid, polytetrafluoroethylene implants.

For citation: Filatova I.A., Shemetov S.A. Combined method of surgical treatment for paralytic lagophthalmos with polytetrafluoroethylene (PTFE) implants. Russian ophthalmological journal. 2018; 11 (2): 27-34.

doi: 10.21516/2072-0076-2018-11-2-27-34 (In Russian).

Conflict of interests: there is no conflict of interests.

Financial disclosure: No author has a financial or property interest in any material or method mentioned.

\section{References}

1. McCord C.D. Eyelid Surgery. New York: Lippincott-Raven; 1995: 99-155.

2. Kasparova E.A., Sobkova O.I.Neurotrophickeratitisonthebackground of paralytic lagophthalmos. Ophthalmology. 2009; 6 (2): 11-4 (in Russian).

3. Kasparov A.A., Kasparova E.A., Sobkova O.I. Treatment of central corneal ulcers on the background of the lagophthalmos using autoconjunctival plastics in combination with optical iridectomy and the outer blefaroplastics. Vestnik oftal'mologii. 2010; 126 (2): 35-7 (in Russian).

4. Zhurova S.G., Brzheskii V.V., Kalinina I.V., Efimova E.L. Treatment of corneal ulcers of kserotic etiology. Klinicheskaja oftal'mologija. 2010; 11 (2): 49-52 (In Russian).

5. Yücel O.E., Artürk N. Botulinum toxin-A-induced protective ptosis in the treatment of lagophthalmos associated with facial paralysis. Ophthal. Plast. Reconstr. Surg. 2012; 28 (4): 256-60. doi:10.1097/ IOP.0b013e31824ee702.

6. Tabashnikova T.V., Serova N.K., Shimanskii V.N., Grigor'eva N.N. The use of botulinum toxin A (lantox) to obtain a temporary druginduced ptosis in neurosurgical patients. Voprosy neirokhirurgii im. N.N. Burdenko. 2012; 4: 43-9 (in Russian).

7. Tabashnikova T.V., Serova N.K., Shimanskii V.N. Treatment of trophic keratopathy and lagophthalmos correction in neurosurgical patients. Oftal'mologicheskie vedomosti. 2013;3:9-16. (in Russian).

8. Zaikova M.V. Ophthalmic Plastic surgery. 2-e izd. Moscow: Meditsina; 1980 (in Russian).

9. Nerobeev A.I., Shurgaya C.M., Marinicheva I.G. Methods of correction of paralytic lagophthalmos. Annals of plastic, reconstructive and aesthetic surgery. 1998; 2: 18-27 (in Russian).

10. Volkov V.V., Brzheskii V.V., Ushakov N.A. Ophthalmic surgery with the use of polymers. Sankt Petersburg: Gippokrat; 2003 (in Russian).

11. Agafonova E.I., Grusha Ya.O. Surgical methods for the treatment of paralytic lagophthalmos. Vestnik oftal'mologii. 2010; 5: 48-52 (in Russian).

12. Skovorodnikova I.V. Method for correction of lagophthalmos in chronic facial paralysis. Patent RF № 2579351; 2016 (in Russian).

13. Pirrello R., D'Arpa S., Moschella F. Static treatment of paralytic lagophthalmos with autogenous tissues. Aesthetic Plast Surg. 2007; 31(6): 725-31. doi:10.1007/s00266-007-0074-7.
14. Ivolgina I.V. Blepharoplasty of lagophthalmos and the extensive ectropion of the lower eyelid in facial paralysis. Novoe $\mathrm{v}$ oftalmologii. 2011; 2: 27-8 (in Russian).

15. Levin R., Shapiro J. Reanimation of the paralyzed eyelid with the enhanced palpebral spring or the gold weight: modern replacements for tarsorrhaphy. Facial Plast. Surg. 2000; 16: 325-36.

16. Bladen J.C., Norris J.H., Malhotra R. Cosmetic comparison of gold weight and platinum chain insertion in primary upper eyelid loading for lagophthalmos. Ophthal. Plast. Reconstr. Surg. 2012; 28 (3): 171-5. doi:10.1097/iop.0b013e3182467bf7.

17. Lessa S., Nanci M., Sebastiá R., Flores E. Treatment of paralytic lagophthalmos with gold weight implants covered by levator aponeurosis. Ophthal. Plast. Reconstr. Surg. 2009; 25 (3): 189-93. doi:10.1097/iop.0b013e3181c0e2e8.

18. Belsare G. Gold weights as upper eyelid implants or facial paralysis. Indian J. Otolaryngol. Head Neck Surg. 2010; 62 (1): 36-9.

19. Grusha Ya.O., Novikov I.A., Agafonova E.I. The first experience of application of the implant for enlarging the upper eyelid with paralytic lagophthalmos. Vestnik oftal'mologii. 2012; 1: 41-3 (in Russian).

20. Chepeha D.B., Yoo J., Birt C., Gilbert R.W., Chen J. Prospective evaluation of eyelid function with gold weight implant and lower eyelid shortening for facial paralysis. Arch. Otolaryngol. Head Neck Surg. 2001; 127 (3): 299-303. doi:10.1001/archotol.127.3.299.

21. Thomas D., Khalifa $Y$. Temporalis fascia in the management of gold eyelid weight extrusion. Ophthal. Plast. Reconstr. Surg. 2005; 21 (2): 153-5. doi:10.1097/01.iop.0000156499.84713.44.

22. Goldberg R.A., Lee S., Jayasundera T., et al. Treatment of lower eyelid retraction by expansion of the lower eyelid with hyaluronic acid gel. Ophthal. Plast. Reconstr. Surg. 2007; 23 (5): 343-8.

23. Mancini R., Taban M., Lowinger A., et al. Use of hyaluronic Acid gel in the management of paralytic lagophthalmos: the hyaluronic Acid gel "gold weight". Ophthal. Plast. Reconstr. Surg. 2009; 25 (1): 23-6. doi:10.1097/IOP.0b013e318192568d.

24. Grusha Ya.O., Ismailova D.S., Ivanchenko Y.F., Agafonova E.I. First experience with the drug stabilized hyaluronic acid for the correction of lagophthalmos. Vestnik oftal'mologii. 2010; 126 (1): 39-42 (in Russian).

25. Dyakov V.E., Dushenkov A.S., Kortunov Yu.A., et al. Implant for reconstructive surgery. Patent RF № 166787; 2003 (in Russian).

Для контактов: Филатова Ирина Анатольевна E-mail: filatova64@yandex.ru 